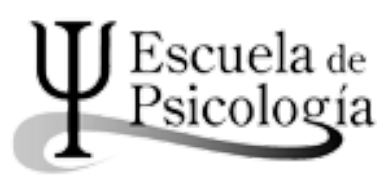

Wímblu, Rev. estudiantes Esc. de psicología, Univ. de Costa Rica. 12 (2): 117-130, 2017 / ISSN: 1659-2107

\title{
Los misterios de la subjetividad: estudio de caso de un paciente con esquizofrenia
}

The mysteries of subjectivity: case study of a patient with schizophrenia

\author{
MSc. Josué Veloz Serrade* \\ Lic. Claudia Cancio-Bello Ayes** \\ Alianys Bejerano Bonilla*** \\ Yalena de la Caridad Cabrera Fernández****
}

Resumen: El objetivo general del presente estudio de caso es caracterizar y analizar desde la perspectiva psicoanalítica, la perspectiva de género y el psicografismo los principales síntomas psicopatológicos que presenta un paciente que acude a consulta desde octubre de 2016. Para realizar la presente investigación se analizaron en profundidad las relatorías de las sesiones del paciente y la expresión pictórica de su subjetividad. El paciente según el DSM 5 presenta un Trastorno F20.9 Esquizofrenia, que pertenece al Espectro de la esquizofrenia y otros trastornos psicóticos, de conjunto con la esquizofrenia converge el Trastorno de Voyeurismo (F65.3).

Palabras claves: psicoanálisis, género, psicografismo y psicosis.

Abstract: The general objective of the present case study is to characterize and analyze, from the psychoanalytic perspective, the gender perspective and psychographics, the main psychopathological symptoms that a patient presents in consultation since October 2016. In order to carry out the present investigation, they were analyzed in depth the rapporteurships of the patient's sessions and the pictorial expression of his subjectivity. The patient according to the DSM 5 presents a disorder F20.9 Schizophrenia, which belongs to the spectrum of schizophrenia and other psychotic disorders, together with schizophrenia converges the Voyeurism Disorder (F65.3).

Key Words: psychoanalysis, gender, psychographics and psychosis.

* Universidad de La Habana. Facultad Latinoamericana de Ciencias Sociales (FLACSO). Cuba. Correo electrónico: josue@,flacso.uh.cu

** Universidad de La Habana. Departamento Ejercicio de la Profesión, Facultad de Psicología. Cuba. Correo electrónico: claudiacancio91@gmail.com

*** Universidad de La Habana. Estudiante de psicología. Cuba.

Correo electrónico: bejeisdi@gmail.com

**** Universidad de La Habana. Estudiante de psicología. Cuba. Correo electrónico: yalenadlacaridad95@gmail.com

Recepción: 6/2/2017 Aceptación: 29/7/2017 


\section{Introducción}

La esquizofrenia está considerada como la afección psicótica de mayor trascendencia médico-social. Constituye la enfermedad psiquiátrica funcional predominantemente endógena, de nivel psicótico e instalación temprana e insidiosa por lo general, que se manifiesta por la ruptura del fenómeno psíquico y la presencia de síntomas en todas sus esferas; entre las que se destacan la disociación ideo-afectivo-conativa. Su evolución es generalmente crónica, con brotes de agudización, y conduce con frecuencia a un deterioro importante de la personalidad. Es la psicosis funcional modelo y se presenta en algún momento de la vida en el $1 \%$ de la población mundial, sin distinción de sexo, color de la piel ni desarrollo socioeconómico, aunque sus manifestaciones clínicas pueden variar de acuerdo con el medio cultural. El cuadro conduce a cambios significativos en la personalidad, lo que se conoce como ruptura en la línea vital, la cual se expresa por importantes cambios en la actividad, volición, intereses, relaciones y hábitos. (González, 2006)

De acuerdo con el DSM-IV (APA, 2000), las esquizofrenias se clasifican en tipo paranoide, desorganizado, catatónico, indiferenciado y residual. De acuerdo con el DSM-IV los criterios diagnósticos están dados por la presencia de ideas delirantes, alucinaciones, lenguaje desorganizado, comportamiento catatónico o gravemente desorganizado, síntomas negativos, por ejemplo, aplanamiento afectivo, alogia o abulia. Desde el inicio de la alteración, una o más áreas importantes de actividad, como son el trabajo, las relaciones interpersonales o el cuidado de uno mismo, están claramente por debajo del nivel previo al inicio del trastorno. Persisten signos continuos de la alteración durante al menos 6 meses. Este período de 6 meses debe incluir al menos 1 mes de síntomas que cumplan con el criterio diagnóstico inicial y puede incluir los períodos de síntomas prodrómicos y residuales. Durante estos períodos prodrómicos o residuales, los signos de la alteración pueden manifestarse sólo por síntomas negativos o por dos o más síntomas antes mencionados, presentes de forma atenuada.

De acuerdo con el DSM-5 (APA, 2014), la esquizofrenia (F20.9) se encuentra formando parte del Espectro de la esquizofrenia y otros trastornos psicóticos. De esta forma se siguen iguales pautas para el diagnóstico, con la diferencia de que se debe especificar el curso de la enfermedad y si no están en contradicción con los criterios de evolución diagnósticos; si hay presencia de catatonía y la gravedad actual del trastorno, aunque se puede realizar el diagnóstico sin referirse a esta última.

El trabajo clínico que se discutirá a continuación está conceptualizado y trabajado desde un modelo de psicoterapia integrativa debido a la valiosa perspectiva que le ofrece al terapeuta. La tarea del clínico integracionista es ofrecer un servicio psicoterapéutico en donde impacten los diversos motivos de consulta de los pacientes tomando como base las fortalezas que potencialmente ofrecen diversos modelos terapéuticos, dado que existen limitaciones inherentes al uso de una sola teoría. No cabe duda de que no todas las terapias son igual de efectivas y que se pueden aplicar diversas técnicas de intervención de forma sistemática, independientemente de las teorías que les dieron base. Sin embargo, es válido aclarar que esto no implica un eclecticismo al azar ni una selección indiscriminada de técnicas. Se trata de un proceso sistemático, resultado de la evaluación de los diferentes niveles y áreas de funcionamiento de cada paciente, los factores y variables particulares y el contexto terapéutico, que incluye el formato o estrategia. La terapia que se utilizó con el paciente $\mathrm{CJ}$, que es el remitente de este artículo, se realizó con base a la integración de las terapias psicológicas provenientes de un acercamiento psicoanalítico desde la mirada de Freud y un análisis desde la perspectiva de género y el psicografismo, teniendo en cuenta las particularidades del sujeto estudiado.

El caso que será presentado, forma parte de la línea de investigación de Depresión y ansiedad del Centro de Orientación y Atención Psicológica Alfonso Bernal del Riesgo (COAP) situado en La Habana, Cuba. El caso es atendido desde octubre de 2016, hasta el momento se han realizado 15 sesiones y se prevé continuar con el trabajo terapéutico. La enfermedad ha ido evolucionando desde hace 20 años aproximadamente, por lo que ha permanecido internado en varias ocasiones en el Hospital Psiquiátrico de La Habana Cdte. Dr. Eduardo Bernabé Ordaz Ducunge. ${ }^{1}$

\section{Desarrollo}

\section{Presentación de Caso}

Paciente hombre de 46 años, de piel blanca, complexión delgada, soltero, no tiene hijos, que vive en la localidad del Cerro con su padre y su hermana mayor. Llega solo a consulta como consecuencia de un delirio hipocondríaco alegando que tenía SIDA, donde se vislumbra la existencia de alucinaciones auditivas al referir que una voz le decía que se hiciera análisis médicos. Saluda a los/las psicólogos/ as tocándolos por un brazo o abrazándolos porque alega que quiere sentir su cariño y que sonríe porque quiere ser agradable Su discurso desorganizado dificulta la comprensión, donde es frecuente la disgregación e incoherencia de pensamiento, llegando a presentar neologismos como "yegual", "compato", entre otros. Presenta disociación ideo-afectivo-conativa, manifestada en delirios no sistematizados, labilidad afectiva, euforia, depresión y alteración de los hábitos higiénicos. El contenido de su delirio generalmente está asociado al daño que 
constantemente le causan las personas a su alrededor, incluyendo hechos del pasado que lo victimizan. También son frecuentes alucinaciones e ilusiones visuales. Constantemente cuenta sueños y chistes, estos últimos de contenido sexual. Hace referencia a personas de su familia como sus tíos, primos, padres y su hermana. Lo que refiere sobre sus tíos y primos, en la mayoría de los casos, tiene una carga emocional negativa; sitúa a su padre en narraciones del presente y a su madre en el pasado; su hermana, por lo general, posee carga afectiva positiva. Posee una vasta cultura, desde conocimientos referentes a pasajes bíblicos, donde no solo alude a los devotos de Jesús, sino también de Jehová; cantantes del ámbito nacional e internacional como Silvio Rodríguez y José José, poesías de diversos artistas, incluyendo el canto de sus temas musicales más conocidos y la declamación de poemas; proverbios iraníes, teoría sobre Psicología, Matemática, donde se destaca una gran habilidad para realizar cálculos matemáticos y relacionar cifras numéricas; la historia de Cuba, personalidades mundialmente reconocidas a lo largo de la historia de la humanidad. En su discurso se repite la referencia a un hombre llamado Paul, el cual funciona como sentenciador, depositario de hechos de carga negativa que lo humillan y lo hacen sentir inferior. Trae a consulta cuadros de su autoría donde se muestran sus ideas fijas (Ver figuras No. 1 y No. 2) con abundancia de colorido o grafito, representando animales, personas, flores, hongos, barcos, peces, etc. Al principio los cuadros constituían regalos para los/las terapeutas, luego pretendía que se vendieran para que se tomaran las ganancias. También trajo una libreta de teléfonos donde había anotado sus descubrimientos sobre la relación entre varias cifras. Comenta que hace orificios en sus cuadros por los "mira huecos" (Ver figuras No. 2 y No. 3) y refiere: "qué lindas las téticas, qué lindos los culitos, el que no ha mirado un huequito no sabe lo que es la vida", por lo que no solo demuestra su impulso a hacerlo, sino que confiesa que lo ha hecho. En el transcurso de las sesiones fue demostrando mayor empatía con los/las terapeutas, mostrando mayor preferencia por el terapeuta hombre. Se denomina a sí mismo "mounstrico" para hacer referencia a sus episodios de agresividad. Indica que es inseguro, se reconoce como diferente de los demás. Repite frases como "la madre de los tomates", "¿verdad?", "¿usted me escucha?",

"Al César lo que es del César, a Dios lo que es de Dios, y a uno lo que es de uno", "sin comentarios", "aplaudo su sinceridad", "les celebro su personalidad", "¿cómo está su familia?", "les ofrezco amistad".

Diagnóstico nosológico:

Esquizofrenia (F20.9).

Voyeurismo (F65.3).
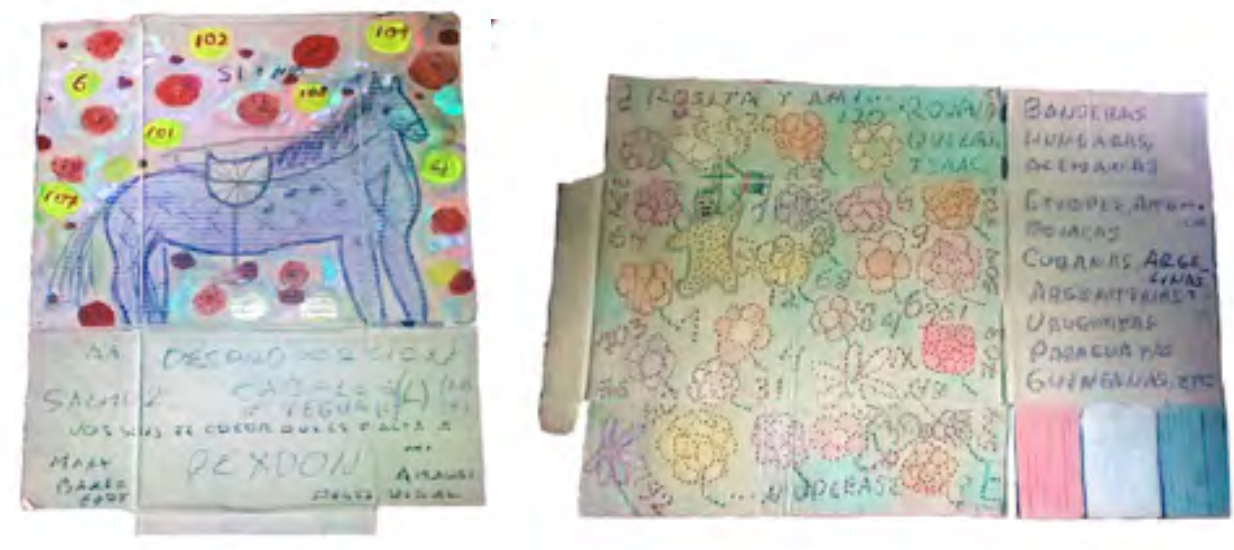

Figura 1: Alianys Bejerano Bonilla

Figura 2: Yalena de la C. Cabrera Fernández

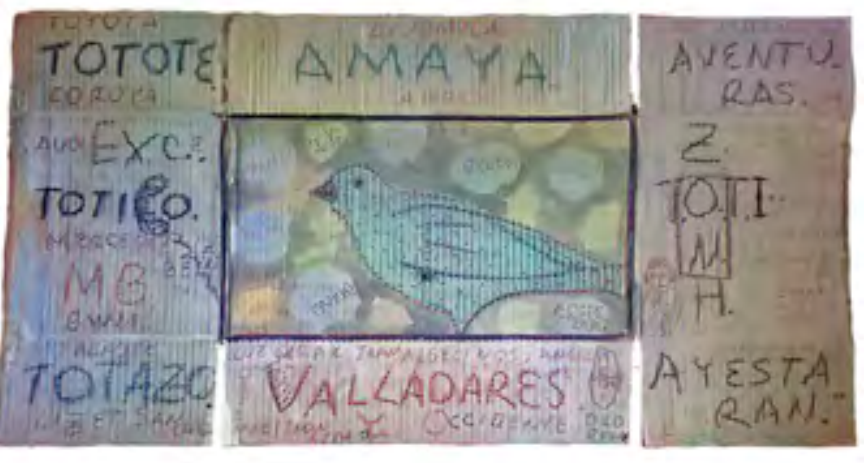

Figura 3: Claudia Cancio-Bello Ayes

\section{Discusión del caso}

A continuación, realizaremos un análisis integrativo del discurso del paciente durante las sesiones, para ello nos apoyaremos en tres enfoques básicos: el pensamiento psicoanalítico, el enfoque de género y la psicografía. En el caso de psicoanálisis, aunque se abordan aspectos más generalmente conocidos, haremos énfasis en la propuesta de le Escuela Grupo Cero del psicoanalista de Miguel Oscar Menassa. Ello no excluye la existencia de otros enfoques, pero en el pensamiento de dicho investigador se da la peculiaridad de entender la estructura del delirio atendiendo a sus grados. 
Es decir, podemos encontrar cortes psicóticos o expresiones delirantes en cualquier padecimiento mental y uno de los elementos de interés en este caso es precisamente la riqueza de expresión de síntomas. Estas valoraciones son el resultado de una intersección entre el pensamiento de Freud y Lacan desde el lugar de la escritura; lo cual abre múltiples posibilidades para la apertura de vías de tratamiento.

Podríamos empezar afirmando que toda enfermedad es social porque toda la enfermedad es el producto de una desavenencia del Yo frente a la realidad, en todos los casos a pedido del Superyo que le dice que la acepte, o a pedido del Ello que le pide que la rechace (Menassa, 1984).

A distinguir la neurosis de la psicosis nos podemos apoyar en el texto, Neurosis y psicosis de Freud, del año 1924. Aquí vamos a encontrar que la neurosis se presenta por un conflicto entre el Yo y el Ello. El Yo no acepta una tendencia libidinosa inconsciente proveniente del Ello, tratando de expulsar y rechazar esa tendencia, por esa vía actúa a favor del Superyo y la realidad objetiva.

El Ello busca las vías por las cuales hacer que esa tendencia inconsciente encuentre gratificación sin que el Yo pueda impedirlo. La reacción que el Yo mostraba antes frente a la tendencia se ejerce ahora sobre el síntoma que es el representante de esa tendencia inconsciente (Freud, 1924).

En la neurosis el síntoma representa la transacción entre el cumplimiento del deseo y su represión, un resultado de la lucha entre el Yo como garante de la ley y al servicio del Superyo y la realidad contra las tendencias inconscientes. En cuanto a la psicosis el conflicto lo encontramos entre la realidad y el Yo. Este frente a un Superyo poco estructurado se pone al servicio del deseo inconsciente. Retoma su libido objetal ubicada en la realidad desapareciendo de su forma habitual y construye otra realidad que irrumpe en el mundo psíquico del paciente, dominada por el principio del placer unido a deseos de carácter incestuoso (Menassa, 1984).

Antes que el psicoanálisis se ocupara de la psicosis los tratamientos de esta se dividían en dos: en uno de ellos se maltrataba al paciente psicótico haciéndosele responsable de todos sus padecimientos; en el otro se le trataba bien haciéndosele irresponsable. En las dos maneras el paciente quedaba aislado. Si se le declaraba culpable se le condenaba a la soledad con lo cual se profundizaba en una de sus dificultades fundamentales: el rechazo primordial de lo otro. Si era inocente entonces se le acompañaba demasiado, con lo cual aumentaba otro de sus problemas: no poder discriminarse del Otro como otro (Menassa, 1988).

El psicoanálisis plantea las cosas del siguiente modo: no habrá tratamiento de la psicosis antes que el paciente establezca un lazo con el analista. Si hay psicoanalista el loco ya no está solo, se puede entablar por esa vía una conversación. Por no faltarle nada al paciente psicótico lo único que se significa en él es el deseo de una madre totipotente y sin fallas, pues es él quien la completa. En el paciente psicótico el Otro no está fuera del cuerpo de la madre, el mismo se mantiene dentro del cuerpo de la madre. Esa unidad con el cuerpo de la madre es lo que el paciente defiende a cualquier costo. Si el sujeto es arrancado de esa unidad pues entonces será sexuado y por ello acontecerá la muerte (Menassa, 1988).

El sujeto en la psicosis concluye o rechaza la mortalidad del ser humano, el Nombre del padre es la posibilidad de metaforizar el deseo de la madre o cambiar la aspiración a la inmortalidad por el Goce. De no ocurrir esto el sujeto psíquico no logra desarrollarse sin la solución psicótica.

Un elemento importante es la confusión que se da en el psicótico entre la palabra y la cosa. En su aparato psíquico en el lugar donde corresponde estar la palabra mesa, él no tiene esa palabra, sino que tiene una mesa. Según Menassa: si el sujeto llegara a decir o a pensar que tiene odio alguien moriría por ese odio. Pero también ocurre cuando yo hablo del deseo inconsciente y alguien puede pesar que le hacen insinuaciones sexuales (Menassa, 1984).

Esta producción delirante está presente en todos ocurre en cualquier sujeto supuestamente normal. La asimilación de una categoría por otra, lo bello por lo divino, lo bueno es lo bello. Esto permite algunos avances en su tratamiento en el sentido de que lo que existe entre las distintas estructuras clínicas es más bien una diferencia de grado en esta equiparación de la cosa y la palabra. Las neurosis por ejemplo están plagadas de cortes psicóticos donde se confunde una explicación con una insinuación, una seducción con una hostilidad (Menassa, 1984).

En el delirio persecutorio, la deformación consiste en una transformación del afecto: aquello que había de ser sentido interiormente como amor es percibido como odio procedente del exterior (también se le conoce con el nombre de proyección). Aquí se aprecia esta confusión entre categorías que referíamos anteriormente de cómo algo puede ser percibido como otra cosa. Evidencia de ello constituye su discurso, marcado por ideas delirantes de daño y perjuicio. Estos se dirigen hacia un hombre llamado Paul, el cual ha constituido en diferentes momentos de la historia de la humanidad, encarnado en diferentes personas y profesiones, personajes reconocidos mundialmente por sus logros y descubrimientos. Ello indica la amenaza que representan para él entes colmados del éxito que él desearía tener. Entre ellos, llama especialmente la atención uno llamado Paul Cézanne (1839-1906), el cual mientras vivió fue un pintor ignorado que trabajó en medio de un gran aislamiento. Además, desconfiaba de los críticos, tenía pocos amigos y, hasta 1895, expuso sólo de forma ocasional. Estaba distanciado incluso de su familia, que tachaba su comportamiento de extraño y no apreciaba el carácter revolucionario de su arte. No es casual la especial similitud que mantiene con $\mathrm{CJ}$.

Sus ideales los constituyen hombres de piel blanca, exitosos profesionalmente en la esfera pública y con las mujeres probablemente, heterosexuales, tal y como dicta la sociedad que debe ser. Creencias como estas pueden traer como 
consecuencia la existencia de rasgos de subvaloración en su personalidad a considerar que su miembro reproductor no es lo suficientemente grande como para ser considerado "viril" en el marco de la sociedad patriarcal donde se vive. En muchas ocasiones, las personas se autodiscriminan, devalúan y culpabilizan por no cumplir adecuadamente con sus deberes de género (Lagarde, 1995). A esto se suma la constante alusión a hechos de su vida donde se ha visto desmoralizado, incapacitado para realizar el acto sexual y frustrado en su relación con las mujeres por las dimensiones de su órgano reproductor ("Carlitos el cañón", "me saqué mi palo de escoba", "voy a sacarme mi pequeño miembro"). En sus cuadros se plasman símbolos fálicos que evidencian su carencia y deseo de revertir la situación. En la figura que encarna a la mujer se evidencia la inhibición de respuesta emocional al emplear solo el grafito como método de pintura y el color morado, indicando angustia (Ver figura No. 4).

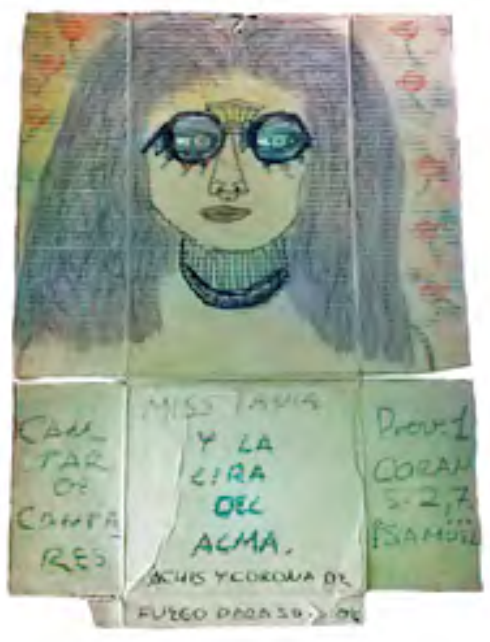

Figura 4: Alianys Bejerano Bonilla

La internalización de atributos que simbolizan la figura masculina, acompañada de una posición de poder y actitudes adoptadas por los hombres, se evidencian en sueños como el del monumento de la universidad. Las muchachas que estaban siendo violadas y él no las defendió por enfrentarse a un hombre que amaneció muerto como consecuencia. La alusión al respeto social que recibió al estar rodeado de mujeres, ante lo cual se siente muy bien; mostrar especial agrado hacia el cuadro del cañón de su autoría, el cual decide dejar en la consulta para dárselo a las psicólogas; el rechazo hacia la psicóloga como figura de poder en la consulta; desempeñar actitudes obscenas que violan la intimidad de las mujeres como parte de la cotidianeidad; ser respetado; cumplir con su palabra; no hablar de lo que no desea. Se observa además su imposibilidad de articular una demanda que muestre un trayecto hacia el deseo de la mujer. No logra realizar la demanda sin agredirla o transgredir límites frente a ella.

Los personajes de alto reconocimiento universal a los que se refiere constantemente $\mathrm{C}$, como Fidel Castro y el Papa, encarnan la figura paterna sublimada, la cual tiene un carácter ambivalente al provocar hechos de carácter tanto positivo como negativo para el paciente. En las observaciones psicoanalíticas sobre un caso de paranoia realizadas por Freud en 1910 (Caso Schreber), el paciente presenta la sublimación del padre encarnado en el símbolo del Sol alegando que lo puede mirar directamente sin que esto dañe sus ojos. La relación entre ambos casos está dada por la relación filial que se mantiene con figuras socialmente inalcanzables.

El Papa cumple una doble función en su discurso, debido a que también constituye un representante de la religión reconocido mundialmente. También es recurrente la referencia a Dios como una figura cercana aunque respeta sobremanera. Muestra así de la concordancia con las concepciones de superioridad genérica, pues no hay gobierno paritario en ningún país. Las iglesias y los partidos políticos son instituciones masculinas y patriarcales: en ellos, las mujeres deben ser feligresía y bases de apoyo respectivamente y aceptar ser conducidas, dirigidas y normadas por hombres. Al referirse a las conductas adoptadas por su madre refleja lo que socialmente se adjudica como el rol de género que deben adoptar las mujeres. Tal es el caso de la asunción de esta figura como visionaria, anclada al espacio privado, de carácter apacible, protector, sumiso. Estas figuras notables vienen a mostrar el escaso espacio ocupado por el Nombre del Padre, su instauración débil y el intento de rellenar esta falla con figuras imposibles o inalcanzables.

El empleo del arte para expresarse, no solo a través de la pintura sino también mediante la declamación de poesías y el canto, permiten comprender su funcionamiento. Los colores que más predominan en sus dibujos son el rojo, el verde y el morado (Ver figuras 5-9), los cuales son muestra de agresividad, ansiedad, agitación y angustia. La manera en la que se distribuyen los elementos en el cuadro es expansiva, lo cual no es característico de un nivel de funcionamiento psicótico; sin embargo, se aprecia la perseveración y disgregación del pensamiento en la repetición de elementos (Ver figuras $1,7,9$ ). Las canciones y poesías que declama tienen un marcado contenido sexual y afectivo, por lo general expresando frustración y duelo por el objeto perdido. 


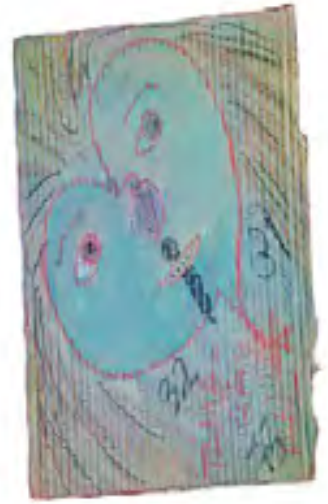

Figura 5: Josué Veloz Serrade

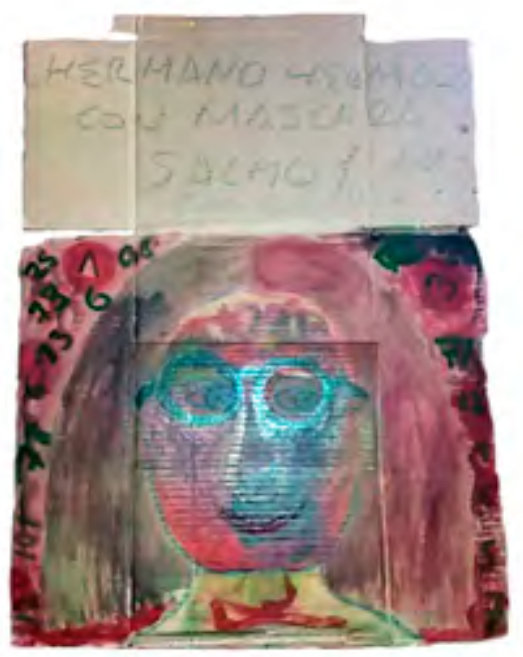

Figura 6: Yalena de la C Cabrera Fernández

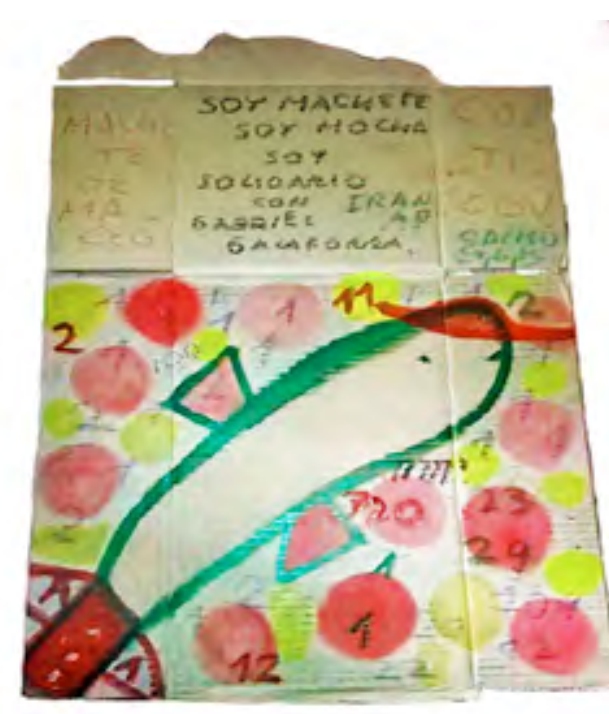

Figura 7: Claudia Cancio-Bello Ayes

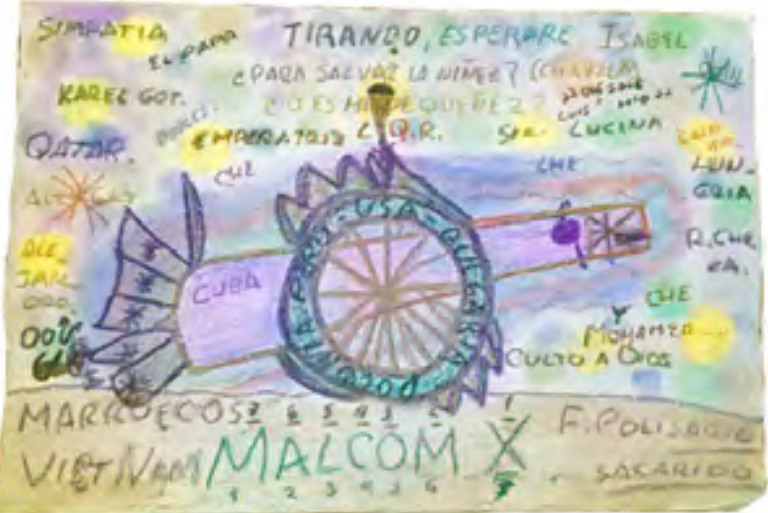

Figura 8: Josué Veloz Serrade

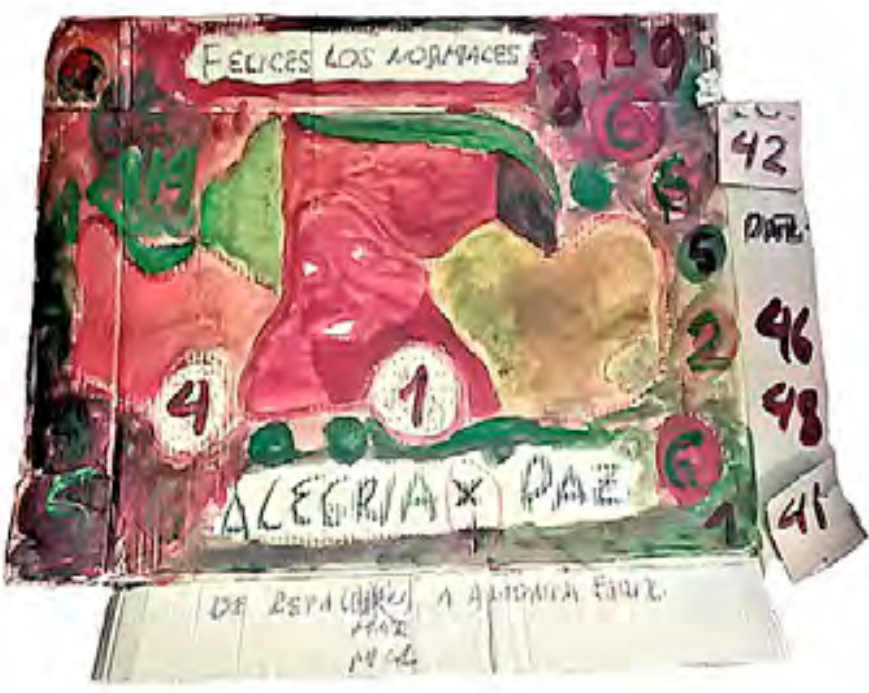

Figura 9: Alianys Bejerano Bonilla 
Criterios que pueden indicar cierto grado de mejoría en el paciente es su referencia al pago hacia los psicólogos, lo cual ocurriría por medio de la venta de sus cuadros. Así mismo, la presencia de actos fallidos en su comportamiento, dados por el olvido de objetos personales en el lugar de consulta que provoca que regrese a buscarlos, contar chistes y sueños.

En su discurso son cotidianas referencias homofóbicas como no querer estar rodeado de "patos", que asesinaron a su madre y la dejaron en manos de las lesbianas, la negación a que Paul lo pisara por atrás, a que Samuel lo "bendijera", y a pintar cuadros de "honguitos" solo con bolitas haciendo referencia a las personas con SIDA por miedo a que la sociedad se indignara. Refiriéndose a este tema, Lagarde (1995) expresa que el sexismo se realiza también en la homofobia, cuando se considera que la heterosexualidad es natural, superior y positiva, y por antagonismo, se supone que la homosexualidad es inferior y es negativa. La homofobia concentra actitudes y acciones hostiles hacia las personas homosexuales. La homofobia encuentra su expresión clarísima cuando horroriza la homosexualidad y se cree que es enfermedad o perversión. También indica: "ojalá no les pese la energía de Paul y me pise por atrás", lo cual se relaciona con la negación de Schreber de perder su virilidad con la frase: iVaya un señor magistrado que se deja j...! aludiendo a la homosexualidad (Freud, Observaciones psicoanalíticas sobre un caso de paranoia. Caso Schreber, 1910).

Desde la perspectiva de género se vislumbra cómo los pacientes en un nivel psicótico de funcionamiento no abandonan sus concepciones machistas producto de la cultura patriarcal donde se desarrollaron y construyeron su concepción del mundo, pudiendo hipotetizar que la repercusión que trae como consecuencia en la subjetividad de los/las sujetos sea la causa de la enfermedad.

Las alusiones al significante falo utilizan significaciones que muestran la vivencia de impotencia y frustración frente a la sexualidad de los otros. No encuentra un modo de simbolizar su demanda de amor que no pase por la apertura de la sexualidad de una manera descarnada y perversa. Interesante que en el paciente no solo se vive la ausencia de un modelo que le permita leer su sexualidad y situarle frente a la Mujer, sino que cuando logra articular algo de su demanda de amor lo hace desde la perversión transgrediendo el lugar del otro en su discurso.

Por otro lado, la figura del padre se divide en un objeto inalcanzable con cualidades extrahumanas por las cuales el sujeto siempre se muestra en un lugar inferior. A su vez el Padre es también dador de la verdad: "él me dijo que estaba enfermo y entonces me hice los análisis". La Madre aparece como lugar de rechazo, esta "no le toma en cuenta" ni acoge su dolor. En otras ocasiones es "dura", "lejana". En la transferencia muestra esa ambivalencia y comienza a ser el garante de aquello que necesita el terapeuta, le provee de cuadros, le pregunta por su familia, se muestra preocupado por su salud, o teme que alguien intente también hacerle daño a él. En otras teme ser abandonado. Se pueden observar en estos elementos sus dificultades para establecer diferenciaciones frente al Otro y al mismo tiempo su dificultad para prescindir de la relación precaria establecida con el terapeuta.

\section{Conclusiones}

Es un paciente de una enfermedad crónica y de larga evolución con un pronóstico reservado.

En el paciente confluyen dos diagnósticos: Esquizofrenia (F20.9) y Voyeurismo (F65.3).

Durante el transcurso de las sesiones se ven elementos que indican avances y retrocesos en el proceso terapéutico.

\section{Notas}

1. Desde el 9 de enero de 1959 se reconoce el Hospital como centro donde el respeto a la dignidad humana de los pacientes mentales constituye la política institucional, así como la introducción de un enfoque científico en el tratamiento médico. A partir de entonces toma posesión de la vocación humanistante Guerrillero, Dr. Eduardo Bernabé Ordaz Ducunge como parte de la en 1992 la de Unidad del Pueblo y para el Pueblo, en 1994 la de Colectivo Moral y en el 2008 el de Unidad de Ciencia y Técnica. Hoy el Hospital Psiquiátrico de La Habana, existe por y para el paciente y se dispone a alcanzar nuevos objetivos de trabajo que eleven a planos superiores los resultados alcanzados.

\section{Referencias bibliográficas}

Alfonso Martínes-Taboas, Guilermo Bernal. (2014). Estudio de casos clínicos. Puerto Rico: Publicaciones Puertorriqueñas, Inc.

American Psychiatric Association. (2013). Diagnostic and statistical manual of mental disorders (5th ed.). Washington, DC: Author

American Psychiatric Association. (2000). Manual diagnóstico y estadístico de los trastornos mentales (4ta ed., text rev.). Ferrer Internacional: Autor

Colectivo de profesores de psiquiatría ISCMH. (1974). Propedéutica y clínica psiquiátrica. La Habana: Pueblo y Educación. 
Freud, Sigmund. (1915). Un caso de paranoia contrario a la teoría psicoanalítica. Versión digital

Freud, Sigmund. (1910). Observaciones psicoanalíticas sobre un caso de paranoia. Caso Schreber. Versión digital

Gónzalez, Ricardo. (2006). Clínica Psiquiátrica Básica Actual. La Habana: Félix Varela.

González, Rafael. (1988). Psiquiatría para médicos generales. Ciudad de la Habana: Científico-Técnica.

González, Rafael. (1980). El médico ante el trastorno Psiquiátrico menor. Santiago de Cuba: Oriente.

Lagarde, Marcela. (1995). Identidad de Género y Derechos Humanos. La construcción de las humanas. Versión digital

Menassa, Miguel Oscar. (1988). Freud y Lacan- Hablados-2. Editorial Grupo Cero. Madrid. 1988.

Menassa, Miguel Oscar. (1984). Neurosis y psicosis. Clase dictada en la Escuela de Psicoanálisis Grupo Cero. Seminario Sigmund Freud el 21 de Mayo de 1984. Revisado en: http://www.extensionuniversitaria.com/num124/p2.htm.

MINSAP. (1997). Glosario Cubano de la 10ma. Revisión de la Clasificación Internacional de Enfermedades Mentales GC-3. Ciudad de La Habana: Hospital Psiquiátrico de La Habana.

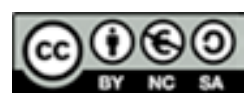

Esta obra está bajo una licencia de Creative Commons Reconocimiento

- NoComercial - CompartirIgual (by-nc-sa) 